\title{
Bestrophin 1 gene analysis and associated clinical findings in a Chinese patient with Best vitelliform macular dystrophy
}

\author{
YING LIN $^{1 *}$, TAO LI $^{1 *}$, HONGBIN GAO $^{2,3^{*}}$, YU LIAN $^{1}$, CHUAN CHEN $^{1,4}$, YI ZHU $^{1,4}$, \\ YONGHAO LI ${ }^{1}$, BINGQIAN LIU ${ }^{1}$, WENLI ZHOU ${ }^{1}$, HONGYE JIANG $^{5}$, XIALIN LIU ${ }^{1}$, \\ XIUJUAN ZHAO ${ }^{1}$, XIAOLING LIANG ${ }^{1}$, CHENJIN JIN $^{1}$, XINHUA HUANG ${ }^{1}$ and LIN LU ${ }^{1}$
}

\begin{abstract}
${ }^{1}$ State Key Laboratory of Ophthalmology, Zhongshan Ophthalmic Center, Sun Yat-sen University, Guangzhou, Guangdong 510060; ${ }^{2}$ Guangdong Laboratory Animals Monitoring Institute, Key Laboratory of Guangdong Laboratory Animals, Guangzhou, Guangdong 510663; ${ }^{3}$ Department of Toxicology, School of Public Health and Tropical Medicine, Southern Medical University, Guangzhou, Guangdong 510515, P.R. China; ${ }^{4}$ Department of Molecular and Cellular Pharmacology, University of Miami Miller School of Medicine, Miami, Fl 33136, USA; ${ }^{5}$ Department of Obstetrics and Gynecology, The First Affiliated Hospital, Sun Yat-sen University, Guangzhou, Guangdong 510000, P.R. China
\end{abstract}

Received October 28, 2016; Accepted June 15, 2017

DOI: $10.3892 / \mathrm{mmr} .2017 .7174$

\begin{abstract}
The aim of the present study was to investigate the clinical characteristics and the underlying genetic causes of Best vitelliform macular dystrophy (BVMD) in a sporadic case in a Chinese patient. A 10 -year-old boy was diagnosed with BVMD; complete ophthalmic examinations were performed, including best-corrected visual acuity, intraocular pressure, slit-lamp examination, fundus photograph, optical coherence tomography and fundus fluorescein angiography imaging. Genomic DNA was extracted from leukocytes of the peripheral blood collected from this patient and his family members. DNA samples from 200 unrelated subjects from the Chinese population were used as controls. A total of 11 exons of the bestrophin 1 (BEST1) gene were amplified by polymerase chain reaction and directly sequenced. The results revealed that the patient presented with yellowish lesions in the macular area. Heterozygous mutations c.292G >A (p.Glu98Lys) in exon 4 and c.1608C $>$ T (p.Thr536Thr) in exon 10 of the BEST1 gene were identified in this sporadic case; however, this was not identified in any of his unaffected family members or in the normal controls. The c.292G >A (p.Glu98Lys) mutation has not been previously reported, whereas the c.1608C $>\mathrm{T}$
\end{abstract}

Correspondence to: Professor Xinhua Huang or Professor Lin Lu, State Key Laboratory of Ophthalmology, Zhongshan Ophthalmic Center, Sun Yat-sen University, 54 Xianlie South Road, Guangzhou, Guangdong 510060, P.R. China

E-mail: 1476557150@qq.com

E-mail: lulin888@126.com

*Contributed equally

Key words: best vitelliform macular dystrophy, bestrophin 1, VMD2, mutation, single nucleotide polymorphism
(p.Thr536Thr) mutation is a previously characterized single nucleotide polymorphism (SNP). In conclusion, BEST1 gene mutations and polymorphisms have been reported in diverse ethnic groups, and the present study identified a novel BEST1 gene mutation and an SNP that occurred simultaneously in a Chinese patient with BVMD.

\section{Introduction}

Best vitelliform macular dystrophy (BVMD), also known as Best disease, is a dominant, bilateral, symmetric, progressive disease of the macular area; patients present with symptoms of metamorphopsia, blurred vision and a decrease in central vision $(1,2)$. BVMD usually has an early onset, often in the first decade of life $(3,4)$; however, there are some atypical types of BVMD (2). It is the second most common form of juvenile macular degeneration and accounts for about $1 \%$ of all cases of macular degeneration (5). At present, it remains an inherited macular dystrophy with no effective treatment.

There are five stages of BVMD that have been described based on fundus presentation: i) Stage 1 (previtelliform stage), which presents normal macula or subtle retinal pigment epithelium (RPE) alterations; ii) Stage 2 (vitelliform stage), in which the macula exhibits a typical well-circumscribed 0.5 -to 2-disc-diameter 'egg-yolk' lesion; iii) Stage 3 (pseudohypopyon stage), in which yellow material accumulates inferiorly to the retina; iv) Stage 4 (vitelliruptive stage), which has a 'scrambled-egg' lesion and partial resorption of the material; and v) Stage 5 (atrophic stage), final macular atrophy that is similar to other macular diseases $(6,7)$. In addition, BVMD may present with or without choroidal neovascularization (8-10).

BVMD is associated with mutations in bestrophin-1 (BEST1; formerly known as the VMD2) (11), which has been mapped to chromosome 11q12-q13. It is $15 \mathrm{~kb}$ in length and contains 11 exons of which 10 are protein-coding (10). At least 269 BEST1 mutations have been reported in BVMD with clinically distinguishable degenerative eye diseases $(12,13)$. 
The bestrophin-1 protein is $68 \mathrm{kDa}$ and contains a highly conserved $\mathrm{N}$-terminal domain that includes several putative transmembrane regions, and a C-terminal domain that is highly variable $(10,14)$. Bestrophin-1 is expressed in the basolateral plasma membrane of the RPE and functions as a calcium-activated chloride channel $(12,15)$. Notably, bestrophin-1 can form a multimer through interaction of the $\mathrm{N}$-terminal and $\mathrm{C}$-terminal domains, which is important for its normal function $(14,16)$. Currently, the pathogenesis of BVMD is still unclear. Identification of BEST1 mutations is the first step in unraveling the pathogenesis of BVMD and may aid genetic counseling. The present study aimed to characterize the clinical presentations of a 10-year old Chinese patient with BVMD and to identify the underlying genetic alterations. The findings from this study expand the mutation spectrum of BESTI and will be valuable for genetic counseling and prenatal diagnosis in patients with BVMD.

\section{Patients and methods}

The 10-year-old male patient in the present study was from the southern area of China with no known familial history of ocular diseases, and was diagnosed with BVMD on July 2015 at Zhongshan Ophthalmic Center (Guangzhou, China). Ophthalmic examinations were performed as follows. Visual acuity was examined using the Early Treatment Diabetic Retinopathy Study (EDTRS) chart (Precision Vision, Woodstock, IL, USA) (17). Anterior segment photograph was taken using a BX 900 slit lamp (Haag-Streit AG, Koeniz, Switzerland). Anterior segment measurements were performed with Pentacam HR version 70700 (Oculus Optikgeräte GmbH, Wetzlar, Germany). Optical Coherence Tomography (OCT) was performed with a Cirrus HD-OCT (Carl Zeiss AG, Oberkochen, Germany). Fundus photography and fundus fluorescein angiography (FFA) imaging were performed using a Heidelberg Retina Angiograph (Heidelberg Engineering GmbH, Heidelberg, Germany) (18-20). Briefly, fluorescein was injected intravenously and stimulated by blue light at a wave length of $465-490 \mathrm{~nm}$. After injection, fluorescein first appeared in the optic nerve and choroid within 8-12 sec (choroidal phase), and then in the arteries 1-3 sec later. Following complete filling of the arteries, the dye began to fill the veins (arteriovenous phase). Following complete filling of the veins (venous phase), roughly $1 \mathrm{~min}$ after injection, the fluorescence began to decrease (recirculation phase). Physical examinations were performed and systemic diseases were excluded.

A total of 200 subjects from the same population without BVMD as well as the unaffected family members of this patient were recruited between March and September, 2015 as controls. Prior to collection of venous blood samples, written informed consent was obtained from all the participating individuals, according to the principles of the Declaration of Helsinki. A total amount of $1 \mathrm{ml}$ of blood sample was collected from each subject, lysed by red blood cell lysis buffer, and centrifuged at 2,000 x $\mathrm{g}$ for $5 \mathrm{~min}$ at room temperature. Genomic DNA was extracted from peripheral blood leucocytes using a DNA extraction kit (Qiagen $\mathrm{GmbH}$, Hilden, Germany) according to the manufacturer's protocol (21-24).

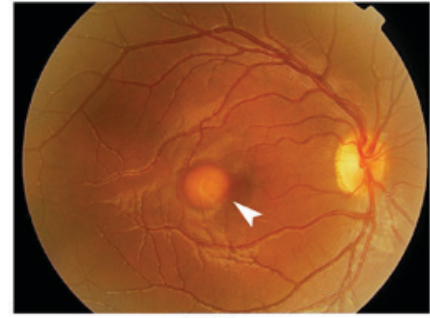

OD

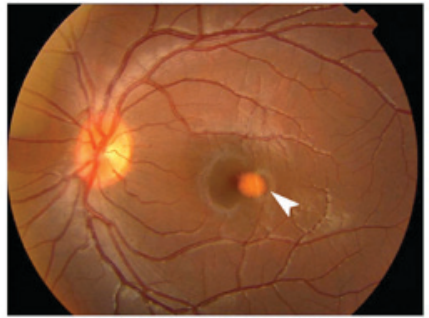

OS
Figure 1. Fundus examination of both eyes. Fundus examination demonstrated abnormal depositions of lipofuscin-like material at the level of the RPE. The classical yellow lesion had an egg yolk appearance (white arrowheads). Magnification $\sim$ x 2.5. OD, oculus dexter (right eye); OS, oculus sinister (left eye).
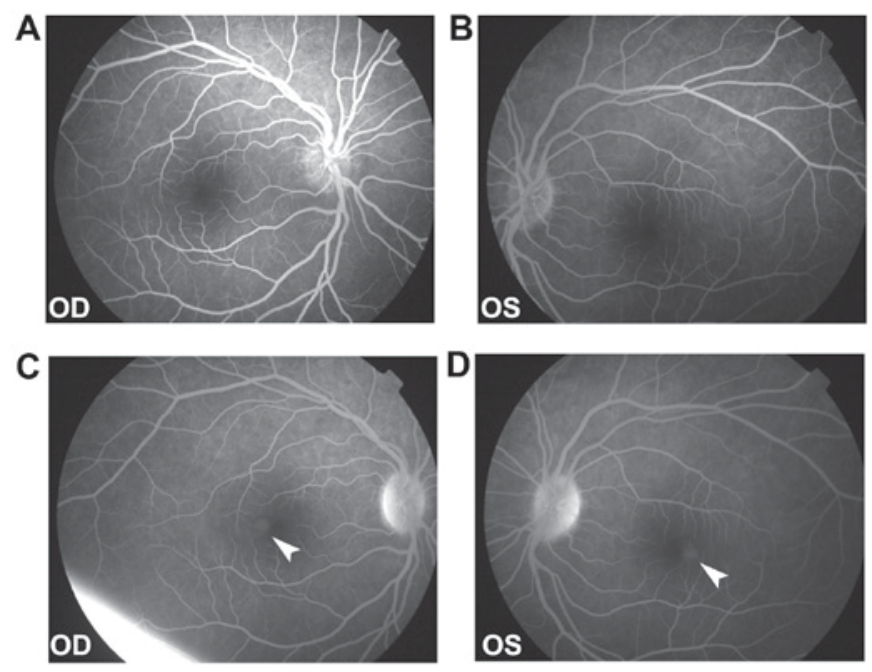

Figure 2. FFA examination of both eyes. (A and B) At the early stage of the angiographic sequence, FFA was normal in both eyes. (C and D) At the late stage of the angiographic sequence, a low amount of hyperfluorescence was observed and was associated with moderate leakage in both eyes (white arrowheads). Magnification $\sim$ x2.5. FFA, fundus fluorescein angiography; $\mathrm{OD}$, oculus dexter (right eye); OS, oculus sinister (left eye).

For mutation detection, exons of the BEST1 gene were amplified by polymerase chain reaction (PCR). The sequences for the forward and reverse primers for PCR were listed in Table I. PCR was conducted in $50 \mu \mathrm{l}$ reactions and the cycling profile included: Initial denaturation at $94^{\circ} \mathrm{C}$ for $5 \mathrm{~min}$, followed by 40 cycles of $94^{\circ} \mathrm{C}$ for $45 \mathrm{sec}, 59^{\circ} \mathrm{C}$ for $45 \mathrm{sec}, 72^{\circ} \mathrm{C}$ for $45 \mathrm{sec}$ and a final extension at $72^{\circ} \mathrm{C}$ for $10 \mathrm{~min}$. All reagents in the PCR reaction were obtained from the PCR amplification kit (Takara Bio, Inc., Otsu, Japan) and used according to the manufacturer's protocols. The PCR products were visualized on a $1 \%$ agarose gel with ethidium bromide staining. After recycling the DNA from the gel using the DNA extraction kit (Takara Bio, Inc.), $20 \mu \mathrm{l}$ PCR product of each subject with a concentration of $100 \mathrm{ng} / \mu \mathrm{l}$ was sequenced by Sanger sequencing method from both directions with an ABI3730 Automated sequencer (Applied Biosystems; Thermo Fisher Scientific, Inc., Waltham, MA, USA). The sequencing primers were the same as the PCR primers (Table I). The sequencing results were analyzed using the SeqManII program of the Lasergene package (DNAStar, Madison, WI, USA), and were compared with the reference sequences (NC_000011.10) 
Table I. Primers for amplification of Bestl exons.

\begin{tabular}{|c|c|c|c|c|}
\hline Exon & Forward (5'-3') & Reverse $\left(5^{\prime}-3^{\prime}\right)$ & $\begin{array}{l}\text { Product } \\
\text { size } \\
\text { (bp) }\end{array}$ & $\begin{array}{c}\text { Annealing } \\
\text { temperature } \\
\left({ }^{\circ} \mathrm{C}\right)\end{array}$ \\
\hline 2 & AGTCTCAGCCATCTCCTCGC & TGGCCTGTCTGGAGCCTG & 212 & 61 \\
\hline 3 & GGGACAGTCTCAGCC ATCTC & CAGCTCCTCGTGATCCTCC & 238 & 58 \\
\hline 4 & AGAAAGCTGGAGGAGCCG & GCGGCAGCCCTGTCTGTAC & 1408 & 59 \\
\hline 5 & GGGGCAGGTGGTGTTCAGA & GGCAGCCTCACCAGCCTAG & 150 & 59 \\
\hline 6 & GGGCAGGTGGTGTTCAGA & CCTTGGTCCTTCTAGCCTCAG & 181 & 59 \\
\hline 7 & CATCCTGATTTCAGGGTTCC & CTCTGGCCATGCCTCCAG & 257 & 59 \\
\hline 8 & AGCTGAGGTTTAAAGGGGGA & TCTCTTTGGGTCCACTTTGG & 215 & 59 \\
\hline 9 & ACATACAAGGTCCTGCCTGG & GCATTAACTAGTGCTATTCTAAGTTCC & 298 & 59 \\
\hline $10 A$ & GGTGTTGGTCCTTTGTCCAC & CTCTGGCATATCCGTCAGGT & 591 & 59 \\
\hline $10 B$ & CTTCAAGTCTGCCCCACTGT & TAGGCTCAGAGCAAGGGAAG & 457 & 59 \\
\hline 11 & CATTTTGGTATTTGAAATGAAGG & CCATTTGATTCAGGCTGTTG & 216 & 59 \\
\hline
\end{tabular}

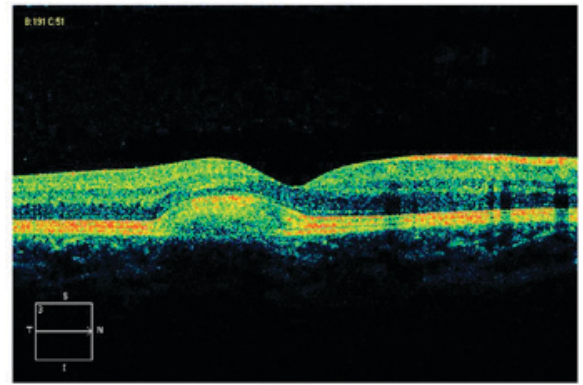

OD

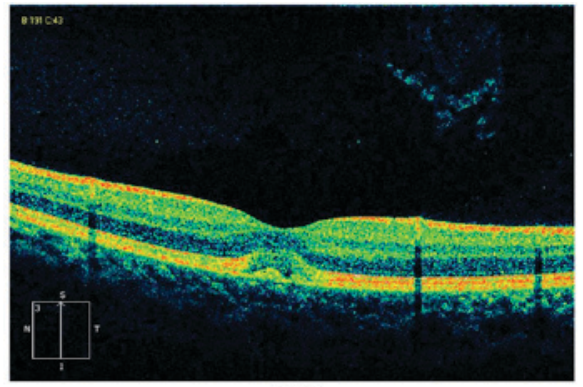

OS

Figure 3. OCT scans of both eyes. The OCT scan printout bears a pseudo-color imaging and retinal mapping is based on different color codes (white, red, orange, yellow, green, blue, and black in order); white being the thickest (High reflectivity, $>470 \mu \mathrm{m})$ and black being the thinnest (low reflectivity, $<150 \mu$ m). Green color indicates intermediate reflectivity. OCT scans revealed that the foveal regions of both eyes were abnormally thick due to neuroretinal detachment from the RPE, which was possibly caused by abnormal accumulation of hyperreflective materials beneath the retina. OCT, optical coherence tomography; RPE, retinal pigment epithelium; OD, oculus dexter (right eye); OS, oculus sinister (left eye).

obtained from the National Center for Biotechnology Information database.

\section{Results}

Refractive error was +6.25 diopter sphere in both eyes with best-corrected visual acuity of 0.2 in the right eye and 0.1 in the left eye; the cornea and the lens were transparent. Fundus examination revealed vitelliruptive lesions with an 'egg-yolk' appearance in both eyes (Fig. 1, white arrowheads). Fluorescein angiography was normal in the early detection period (within 1 min of fluorescein injection, Fig. 2A and B) and there was a small amount of hyperfluorescence of the angiographic sequence with moderate leakage at the late detection period (after 10 mins of fluorescein injection, Fig. 2C and D, white arrowheads) in both eyes.

OCT scans revealed that the foveal region of both eyes was abnormally thick due to neuroretinal detachment from the RPE (Fig. 3), which was probably triggered by the abnormal accumulation of hyper-reflective materials beneath the retina of both eyes.

Sequence analysis identified two heterozygous genetic variations in this patient: c.292G >A (p.Glu98Lys) in exon
4 and c.1608C $>$ T (p.Thr536Thr) in exon 10, (Fig. 4). Neither of these variations were identified in the unaffected family members or normal controls from the same population. The c.1608C >T (p.Thr536Thr) mutation in BEST1 is a previously identified single nucleotide polymorphisms (SNP) (4), whereas c.292G >A (p.Glu98Lys) is a novel mutation.

\section{Discussion}

BVMD is an autosomal dominant disease characterized by egg yolk-like lesions in the macula (5). During the early stages of the disease, patients with BVMD present with abnormal depositions of lipofuscin-like material at the RPE layer that classically resembles an egg yolk $(16,25)$. In the present study, a 10-year-old patient was in early stage 2 of BVMD. Accumulation of lipofuscin-like material in the RPE was observed, although his visual acuity was normal. As pathology of the disease progresses, vision loss may occur if RPE disorganization eventually undermines the overlying photoreceptors. Patients may also exhibit multiple extramacular lesions, hemorrhage, macular holes, or glaucoma $(7,26,27)$. It should be noted that the patient in the present study exhibited high hyperopia at the age of 10 , 


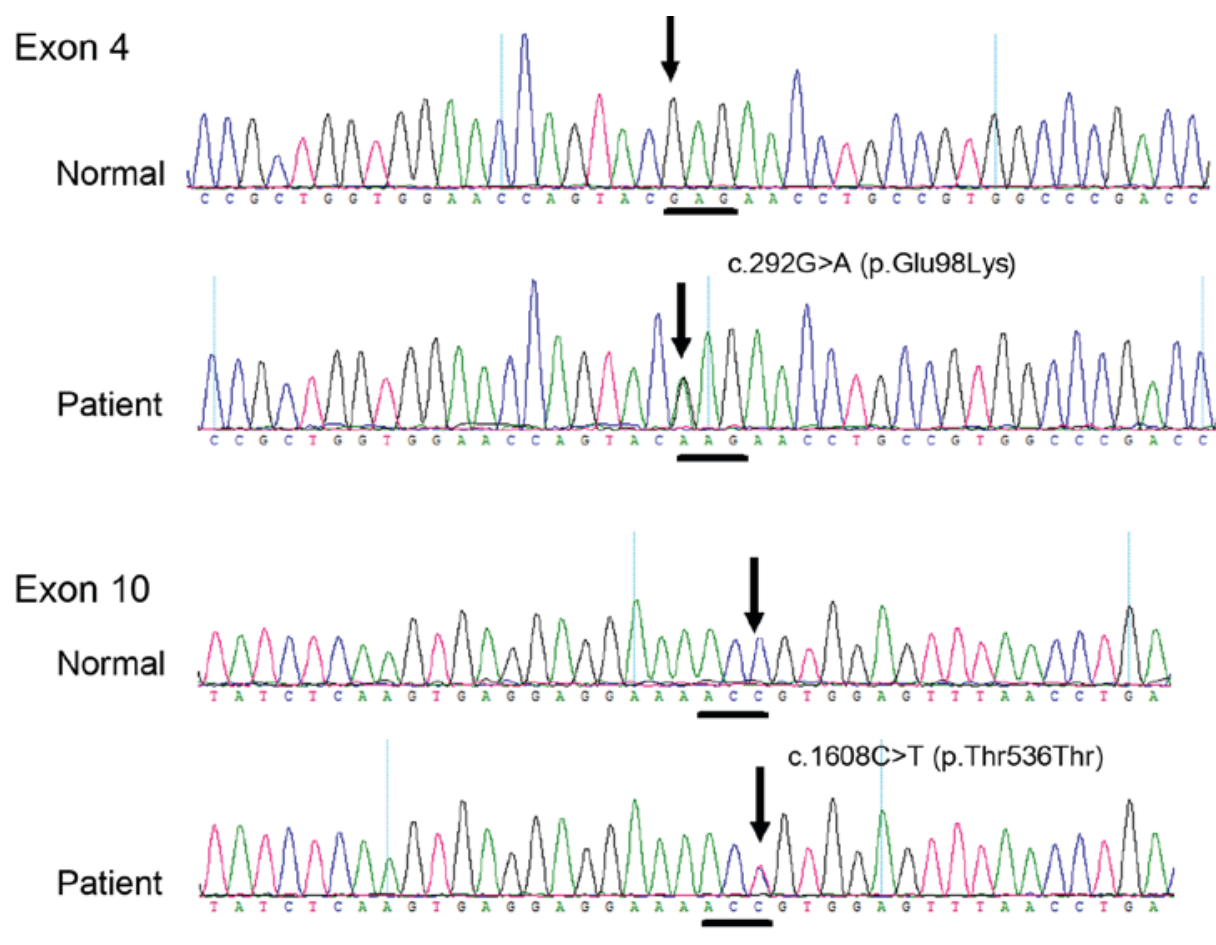

Figure 4. Sequence analysis of this patient and controls. Two heterozygous mutations, including c.292G $>$ A (p.Glu98Lys) in exon 4 and c.1608C $>$ T (p.Thr536Thr) in exon 10, were identified in the BVMD patient, but not in any of the unaffected family members or the normal controls in the same population. BVMD, Best vitelliform macular dystrophy.

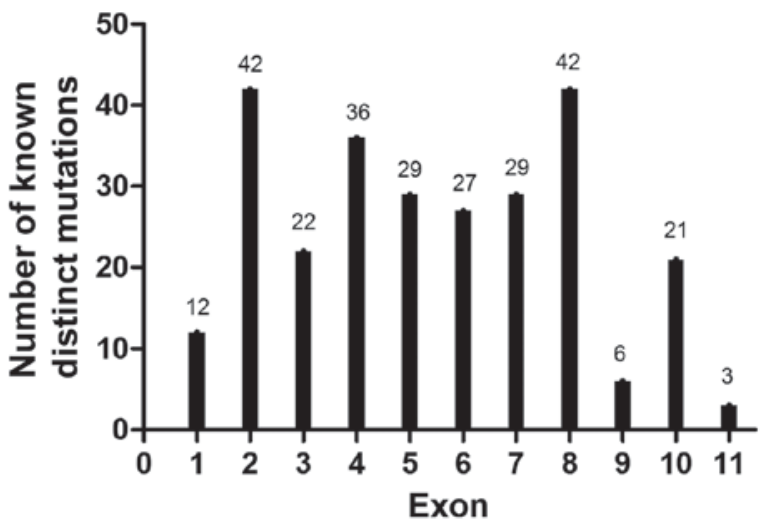

Figure 5. Distribution of the known distinct mutations identified in the BEST1 gene.

which was similar to the reports of other studies of BVMD patients $(7,27)$.

$\mathrm{BVMD}$ is associated with mutations in the BESTI gene. To date, a total number of 269 unique BESTI DNA variants have been identified and are listed in the Regensburg University database (http://www-huge.uni-regensburg.de/BEST1_database/). An overview of the distribution of the known distinct exonic mutations is provided in the Fig. 5; these data appear to indicate that exons 2, 4 and 8 are hot spots in the BESTI gene, as $>30$ mutations have been reported among these exons so far. Consistently, the c.292G $>$ A (p.Glu98Lys) mutation identified in the present study is also located in exon 4 . The c. $1608 \mathrm{C}>\mathrm{T}$ (p.Thr536 Thr) mutation identified in this patient is a known SNP. Various other SNPs have been identified in the BEST1 gene, including c.87C >T (p.Tyr29Tyr) and c.109T >C
(p.Leu37Leu) in exon 2, c.219C >A (p.Ile73Ile) in exon 3, and c.1410G $>$ A (p.Thr470Thr), c.1557C $>$ T (p.Ser519Ser) and c.1608T $>C$ (p.Thr536Thr) in exon $10(4,28)$. The cellular function of the bestrophin-1 has not been fully elucidated. Therefore, it remains challenging to predict which mutations may be pathological.

Many of the disease-causing mutations in BESTl are dominant negative and affect the chloride channel function of the protein (29). These mutations cause amino acid alterations in the highly acidic region of the C-terminus, disrupt the interaction between the $\mathrm{N}$ - and the $\mathrm{C}$-terminal domains and may interfere with the formation of the bestrophin multimer (16). A previous study reported that patients with BVMD may have two possible disease-causing variations in BEST1, neither of which causes macular disease alone (11). It remains unclear how the two mutations act in unison and affect protein function; it is possible that multiple allele abnormalities may be required for the pathogenesis of the disease. Understanding the underlying mechanisms of how BEST1 mutations cause clinical presentation and the characterization of BVMD may greatly improve the diagnosis and treatment of these patients in the future.

A limitation of the present study is that only one patient with BVMD was examined. Future studies are required to investigate independent cohorts of patients affected by BVMD and to characterize the association between different mutations and clinical phenotypes.

In conclusion, the present study identified one novel mutation and one SNP in BESTI in a Chinese patient with BVMD. These findings expand the mutation spectrum of BEST1 and will be valuable for genetic counseling and prenatal diagnosis in patients with BVMD. 


\section{Acknowledgements}

The authors thank the patient and his family, as well as the control volunteers for participating in this study. The present study was supported by The National Natural Science Foundation of China (grant nos. 81500709, 81570862 and 81670872), and the State Scholarship Fund from the China Scholarship Council (CSC).

\section{References}

1. Pagon RA, Adam MP, Ardinger HH, Wallace SE, Amemiya A, Bean LJH, Bird TD, Ledbetter N, Mefford HC, Smith RJH and Stephens K: Best vitelliform macular dystrophy. GeneReviews(®), 1993.

2. Marmorstein AD, Kinnick TR, Stanton JB, Johnson AA, Lynch RM and Marmorstein LY: Bestrophin-1 influences transepithelial electrical properties and $\mathrm{Ca} 2+$ signaling in human retinal pigment epithelium. Mol Vis 21: 347-359, 2015.

3. Liu J, Zhang Y, Xuan Y, Liu W and Wang M: Novel BEST1 mutations and special clinical features of best vitelliform macular dystrophy. Ophthalmic Res 56: 178-185, 2016.

4. Caldwell GM, Kakuk LE, Griesinger IB, Simpson SA, Nowak NJ, Small KW, Maumenee IH, Rosenfeld PJ, Sieving PA, Shows TB and Ayyagari R: Bestrophin gene mutations in patients with Best vitelliform macular dystrophy. Genomics 58: 98-101, 1999.

5. Marchant D, Yu K, Bigot K, Roche O, Germain A, Bonneau D, Drouin-Garraud V, Schorderet DF, Munier F, Schmidt D, et al: New VMD2 gene mutations identified in patients affected by Best vitelliform macular dystrophy. J Med Genet 44: e70, 2007

6. Lin Y, Gao H, Liu Y, Liang X, Liu X, Wang Z, Zhang W, Chen J, Lin Z, Huang $X$ and Liu Y: Two novel mutations in the bestrophin-1 gene and associated clinical observations in patients with best vitelliform macular dystrophy. Mol Med Rep 12: 2584-2588, 2015 .

7. Wittström E, Ponjavic V, Bondeson ML and Andréasson S: Anterior segment abnormalities and angle-closure glaucoma in a family with a mutation in the BEST1 gene and Best vitelliform macular dystrophy. Ophthalmic Genet 32: 217-227, 2011.

8. Lorenz B and Preising MN: Best's disease. Overview of pathology and its causes. Ophthalmologe 102: 111-115, 2005 (In German)

9. Parodi MB, Zucchiatti I, Fasce F and Bandello F: Bilateral choroidal excavation in best vitelliform macular dystrophy. Ophthalmic Surg Lasers Imaging Retina 45: e8-e10, 2014.

10. Nourinia R, Roshandel D, Lima BS and Sayanjali S: Best disease associated with macular hole. Retin Cases Brief Rep 9: 7-12, 2015.

11. Kinnick TR, Mullins RF, Dev S, Leys M, Mackey DA, Kay CN, Lam BL, Fishman GA, Traboulsi E, Iezzi R and Stone EM: Autosomal recessive vitelliform macular dystrophy in a large cohort of vitelliform macular dystrophy patients. Retina 31: 581-595, 2011.

12. Lee CS, Jun I, Choi SI, Lee JH, Lee MG, Lee SC and Kim EK: A novel BEST1 mutation in autosomal recessive bestrophinopathy. Invest Ophthalmol Vis Sci 56: 8141-8150, 2015.

13. Johnson AA, Bachman LA, Gilles BJ, Cross SD, Stelzig KE, Resch ZT, Marmorstein LY, Pulido JS and Marmorstein AD Autosomal recessive bestrophinopathy is not associated with the loss of bestrophin-1 anion channel function in a patient with a novel BEST1 mutation. Invest Ophthalmol Vis Sci 56: 4619-4630, 2015
14. Tsunenari T, Sun H, Williams J, Cahill H, Smallwood P, Yau KW and Nathans J: Structure-function analysis of the bestrophin family of anion channels. J Biol Chem 278: 41114-41125, 2003.

15. Mladenova K, Petrova SD, Andreeva TD, Moskova-Doumanova V, Topouzova-Hristova T, Kalvachev Y, Balashev K, Bhattacharya SS, Chakarova C, Lalchev Z and Doumanov JA: Effects of $\mathrm{Ca} 2+$ ions on bestrophin-1 surface films. Colloids Surf B Biointerfaces 149: 226-232, 2017.

16. Marmorstein AD, Marmorstein LY, Rayborn M, Wang X, Hollyfield JG and Petrukhin K: Bestrophin, the product of the Best vitelliform macular dystrophy gene (VMD2), localizes to the basolateral plasma membrane of the retinal pigment epithelium. Proc Natl Acad Sci USA 97: 12758-12763, 2000.

17. Xi L, Liu Y, Zhao F, Chen C and Cheng B: Analysis of glistenings in hydrophobic acrylic intraocular lenses on visual performance. Int J Ophthalmol 7: 446-451, 2014.

18. Abràmoff MD, Garvin $\mathrm{MK}$ and Sonka M: Retinal imaging and image analysis. IEEE Rev Biomed Eng 3: 169-208, 2010.

19. Tan X, Zhan J, Zhu Y, Cao J, Wang L, Liu S, Wang Y, Liu Z, Qin Y, Wu M, et al: Improvement of uveal and capsular biocompatibility of hydrophobic acrylic intraocular lens by surface grafting with 2-methacryloyloxyethyl phosphorylcholine-methacrylic acid copolymer. Sci Rep 7: 40462, 2017.

20. Spaide RF, Klancnik JM Jr and Cooney MJ: Retinal vascular layers imaged by fluorescein angiography and optical coherence tomography angiography. JAMA Ophthalmol 133: 45-50, 2015.

21. Lin Y, Ai S, Chen C, Liu X, Luo L, Ye S, Liang X, Zhu Y, Yang $\mathrm{H}$ and Liu Y: Ala344Pro mutation in the FGFR2 gene and related clinical findings in one Chinese family with Crouzon syndrome. Mol Vis 18: 1278-1282, 2012.

22. Lin Y, Liang X, Ai S, Chen C, Liu X, Luo L, Ye S, Li B, Liu Y and Yang H: FGFR2 molecular analysis and related clinical findings in one Chinese family with Crouzon syndrome. Mol Vis 18: 449-454, 2012.

23. Lin Y, Liu X, Yu S, Luo L, Liang X, Wang Z, Chen C, Zhu Y, Ye S, Yan H and Liu Y: PAX6 analysis of two sporadic patients from southern China with classic aniridia. Mol Vis 18: 2190-2194, 2012.

24. Zhang Y, Morgan R, Chen C, Cai Y, Clark E, Khan WN, Shin SU, Cho HM, Al Bayati A, Pimentel A and Rosenblatt JD: Mammary-tumor-educated B cells acquire LAP/TGF- $\beta$ and PD-L1 expression and suppress anti-tumor immune responses. Int Immunol 28: 423-433, 2016.

25. Uggenti C, Briant K, Streit AK, Thomson S, Koay YH, Baines RA, Swanton E and Manson FD: Restoration of mutant bestrophin-1 expression, localisation and function in a polarised epithelial cell model. Dis Model Mech 9: 1317-1328, 2016.

26. Liu J, Yi X, Zhang Y, Wei L and Xu G: Bilateral macular holes and a new onset vitelliform lesion in Best disease. Ophthalmic Genet 38: 79-82, 2017.

27. Ponjavic V, Eksandh L, Andréasson S, Sjöström K, Bakall B, Ingvast $\mathrm{S}$, Wadelius $\mathrm{C}$ and Ehinger $\mathrm{B}$ : Clinical expression of Best's vitelliform macular dystrophy in Swedish families with mutations in the bestrophin gene. Ophthalmic Genet 20: 251-257, 1999.

28. Allikmets R, Seddon JM, Bernstein PS, Hutchinson A, Atkinson A, Sharma S, Gerrard B, Li W, Metzker ML, Wadelius C, et al: Evaluation of the best disease gene in patients with age-related macular degeneration and other maculopathies. Hum Genet 104: 449-453, 1999.

29. Reichhart N, Milenkovic VM, Halsband CA, Cordeiro S and Strauss O: Effect of bestrophin-1 on L-type Ca2+ channel activity depends on the $\mathrm{Ca} 2+$ channel beta-subunit. Exp Eye Res 91: 630-639, 2010. 